\title{
On the Effects of Entry under Flexible Production Techniques: An Example of Quasi-Anticompetitiveness
}

\author{
Emanuele Bacchiega* \\ Paolo G. Garella ${ }^{\dagger}$ \\ University of Bologna \\ University of Milano
}

January 2007

\begin{abstract}
We study reactions to entry in a Cournot model, contrasting the case where firms are endowed with unchangeable technologies against that where technologies are flexible. By the latter we mean that firms can change the installed production technique at zero cost (fully flexible technologies). We show that when firms are technologically flexible, entry can increase equilibrium prices. The analysis is cast in a shortrun time horizon to simplify exposition, but its predictive power may better relate to the long run.
\end{abstract}

Keywords: Entry, Technological Flexibility, Oligopoly Theory.

JEL: D43, L1, L11, L13.

*bacchieg@spbo.unibo.it, Dipartimento di Scienze Economiche, Piazza Scaravilli 2, 40126 - Bologna, Italy.

${ }^{\dagger}$ paolo.garella@unimi.it, Dipartimento di Scienze Economiche, Aziendali e Statistiche, via Conservatorio 7, 20122 - Milano, Italy. The author acknowledges support by the University of Crete, under Marie Curie Transfer of Knowledge Fellowship-EC's 6th Framework Programme, n. MTKD-CT-014288. 


\section{Introduction}

The pro-consumer effects of entry are generally associated to the prediction that industry price is likely to decrease as the number of competitors increases. Formally, in the study of Cournot oligopoly, this property is referred to as "quasi-competitiveness". Its opposite, namely quasianticompetitiveness, represents the counter-intuitive situation of markets with prices increasing in the number of competitors.

The conditions for quasi-competitiveness are an object of analysis since MacManus (1962) and (1964). Thereafter, Frank (1965), Ruffin (1971), Okuguchi (1973), Seade (1980) and Amir and Lambson (2000) work out -by means of various analytical tools- the conditions for quasi-competitiveness. ${ }^{1}$ In particular, Amir and Lambson (2000) find a quite simple condition, related to the shapes of the (inverse) demand function and of the firms' cost function, that guarantees quasi-competitiveness or that leads to its opposite.

So far, the assumption that the technologies available to the producers are assigned at the outset of the analysis and cannot be changed has been maintained. In this paper we provide a simple example of an entry model in which a firm active in the industry may react to entry through the usual adjustment in the output level but also through the substitution of the production technology with another one, better tailored to the new competitive conditions. We show that in this case Cournot competition can display "quasi-anticompetitiveness".

In the example, the possibility to change the production technology proves to be crucial for obtaining an increase in the market price following the shift from a monopoly to a duopoly regime. This point makes our approach depart from the quoted literature. If firms are allowed to react in a broader manner to the entry of rivals, then the comparisons between cost and demand functions represent a part only of the analysis necessary to assess the properties of industry equilibria. Indeed one has to ascertain which cost functions will be selected by strategic agents in response to entry.

We shall therefore contrast our assumption of firms displaying "technical

\footnotetext{
${ }^{1}$ Moreover they unveil the tight relations between quasi-competitiveness, uniqueness and stability of Cournot-Nash equilibria.
} 
flexibility" with the case, usually treated in the literature, of firms characterized by "technical inflexibility". In particular, we have in mind a situation in which a firm can choose among a technology that implies low cost for large output levels, and one leading to lower production costs at low output levels.

The paper is organized as follows: Section 2 describes the model, section 3 develops the equilibrium analysis, Section 4 briefly concludes.

\section{The Model}

We start by describing the technology side of the model. A production plan for firm $i$, is a vector, $\mathbf{y}_{i} \in \Re^{2}$, defined as $\mathbf{y}_{i}=(z, q)$, where $z \leq 0$ represents the quantity of input and $q$ represents output. $Y$ denotes a generic production set, namely a collection of production plans. Two production sets are potentially available to the entrepreneurs. The first production set is defined as follows:

$$
Y^{K}=\{(z, q): z \leq-1\} \cup\{(z, q): z \leq 0 \cap q \leq 0\} .
$$

Clearly the number -1 in the condition $z \leq-1$ could be replaced by any negative real number. The second production set is defined as

$$
Y^{V}=\{(z, q): z \leq 0, q-\beta \sqrt{-z} \leq 0\},
$$

with $\beta>0$.

The two production sets can be thought of as deriving from two ways of combining the same input. The first requires at least one unit of input in order to get any desired positive production level, with increasing returns to scale, and it allows inactivity at zero cost. The second, which implies no minimum input, displays decreasing returns to scale. Let $\mathcal{T}_{i}$ be the collection of production sets available to a generic firm $i$, and denote with $T_{i}$ the generic element of $\mathcal{T}_{i}$. Then we assume $\mathcal{T}_{i}=\left\{Y^{K}, Y^{V}\right\}$ for any active firm. We define technology choice by a firm as the selection of one element from the set $\mathcal{T}_{i}$. This choice determines the firm's production costs, which are straightforwardly derived. Formally, for each technology, there exists a minimum quantity of input necessary to produce the output level $q_{i}$, denoted 
$z\left(T_{i}, q_{i}\right)$. Clearly, $z\left(Y^{K}, q_{i}\right)=-1$, and $z\left(Y^{V}, q_{i}\right)=-\left(1 / \beta^{2}\right) q_{i}^{2}$. In case $Y^{K}$ is chosen the total cost borne for producing any quantity is $C_{K}=F$, where $F=\hat{w}$ and $\hat{w}$ is the (constant) unit price of input. In case $Y^{V}$ is selected the cost function writes $C_{V}(q)=(c / 2) q^{2}$, with $c=2\left(\hat{w} / \beta^{2}\right)$. It is worth to remark that neither technology is more efficient than the other. Their relative efficiency depends on the firm's production scale, with $Y^{K}$ leading to lower total cost for large production levels and $Y^{V}$ for low levels. By contrast, $Y^{K}$ implies increasing, while $Y^{V}$ constant (and nil), marginal costs.

On the consumption side, we adopt the linear specification of inverse market demand for a homogeneous commodity:

$$
p(q)=a-b q,
$$

where $p$ denotes the market price and $q$ is the total quantity produced, $a$ and $b$ are, as usual, positive constants.

Notice that if both $Y^{K}$ and $Y^{V}$ belong to $\mathcal{T}_{i}$, then ex-ante firm $i$ is endowed with a production set which is $Y^{K} \cup Y^{V}$. At the production stage, however, once the choice over $\mathcal{T}_{i}$ has been made, the production set reduces to the one that has been chosen. A firm first chooses its technology, then chooses its production plan, $\mathbf{y}_{i}$, aiming at maximizing profits. Formally, letting $\hat{T}_{i} \in \mathcal{T}_{i}$ denote the technology chosen by firm $i$, firm $i$ 's output choice problem is

$$
\max _{\mathbf{y}_{i} \in \hat{T}_{i}} \pi_{i}\left(\mathbf{y}_{i}\right)
$$

where $\pi_{i}(\cdot)$ denotes the profit function $\pi_{i}\left(\mathbf{y}_{i}\right)=p(q) q_{i}-\hat{w} z\left(\hat{T}_{i}, q_{i}\right)$. Letting $\mathbf{y}\left(\hat{T}_{i}\right)$ denote the solution to problem (1) for firm $i$, technology choice is given by the solution to

$$
\max _{T_{i} \in \mathcal{T}_{i}} \pi_{i}\left(\mathbf{y}\left(T_{i}\right)\right) \text {. }
$$

In the ensuing analysis we shall indifferently refer to the production set $Y^{K}$ or to "technology $K$ " and to the production set $Y^{V}$ or to "technology $V$ ". We shall also adopt the standard techniques of profit maximization with respect to output, $q_{i}$, for a given cost function. 


\section{Equilibrium Analysis}

We shall perform equilibrium analysis treating first the optimal choices of a monopolist and then moving to the subgame-perfect Nash equilibrium of a symmetric duopoly. The comparison between the two market structures will shed light on the quasi-anticompetitiveness of the model, as one can juxtapose the two examples by considering duopoly as deriving from entry by a new competitor into a monopolized market. In particular we will show that there exist non-degenerate parametric configurations under which a monopolist chooses technology $K$, while two duopolists choose technology $V$. Hence, entry by a second firm, under full flexibility, leads to a change in technology by the former monopolist and finally to an increase in the equilibrium price.

\subsection{Monopoly}

Assume that a single firm is active on the market, label it $M$ and exclude the threat of entry. If the firm selects the $K$-technology for production, the solution to problem (1) yields a profit-maximizing quantity, $q_{M}^{K}$, and unit price, $p_{M}^{K}$, given as $q_{M}^{K}=a /(2 b)$ and $p_{M}^{K}=a / 2$; the profit level, $\pi_{M}\left(q_{M}^{K}\right)$, is

$$
\pi_{M}\left(q_{M}^{K}\right)=\frac{a^{2}}{4 b}-F
$$

Similarly, if it selects the $V$ technology, optimal quantity and market price are $q_{M}^{V}=a /(2 b+c)$ and $p_{M}^{V}=(b+c) q_{M}^{V}$, with associated profits

$$
\pi_{M}\left(q_{M}^{V}\right)=\frac{a^{2}}{4 b+2 c}
$$

Letting $a^{2} c /\left(8 b^{2}+4 b c\right) \equiv F^{I I}$, direct comparison between (3) and (4) shows that

$$
\pi_{M}\left(q_{M}^{K}\right)>\pi_{M}\left(q_{M}^{V}\right) \Leftrightarrow F<F^{I I} .
$$

We can then state the following

Lemma 1 (i) If $F<F^{I I}$ the monopolist chooses technology $K$ and market price is $p_{M}^{K}=\frac{a}{2}$. 
(ii) If $a^{2} /(4 b)>F>F^{I I}$ the monopolist chooses technology $V$ and market price is $p_{M}^{V}=\frac{a(b+c)}{2 b+c}$.

\subsection{Duopoly}

Assume now that another firm enters the market. From now on we shall label the "incumbent" -former monopolist- firm 1 and the "entrant" firm 2. In response to the new competitive framework, firm 1 modifies the quantity supplied to the market and -eventually- changes the technology chosen for producing it. Similarly, firm 2 selects its own technology from the set $\mathcal{T}_{2}$ and then sets quantity taking into account its opponent's decisions; in other words both firms will solve strategically problem (1).

In order to formally study the strategic interaction between firms we setup a simultaneous-move two-stage game, $\Gamma$, in which firms 1 and 2 first select which technology to use and then choose the quantity to produce, aiming at profit maximization. For the purpose of our analysis we first focus on the conditions leading to the specific symmetric duopoly equilibrium in which both firms select the decreasing returns to scale technology $V .{ }^{2}$ If both firms at the first stage select the production set $Y^{V}$, then Cournot equilibrium quantities and price are ${ }^{3}$

$$
q_{i}^{V V}=\frac{a}{3 b+c} \text { and } p^{V V}=\frac{a(b+c)}{3 b+c}, \text { for } i=1,2
$$

with corresponding equilibrium profits

$$
\pi_{i}^{V V}=\frac{a^{2}(2 b+c)}{2(3 b+c)^{2}}
$$

Both firms choosing $V$ at the first stage and then setting quantity $a /(3 b+c)$ is a SPNE of $\Gamma$ if there are no advantageous unilateral deviations from this strategy for one firm. In order to check this consider the case in which firm 1 chooses the $K$ technology while firm 2 still selects the $V$ one. In this case

\footnotetext{
${ }^{2}$ It is quite superfluous, for the purposes of this paper, to provide a full taxonomy of the possible (subgame-perfect) Cournot-Nash equilibria of the game $\Gamma$. For a brief discussion on this issue see the comments after Lemma 2 on page 7.

${ }^{3}$ The notation $V V$ stands for "firm 1 chooses technology $V$ and firm 2 chooses technology $V^{\prime \prime}$.
} 
firm's quantities and industry price write as ${ }^{4}$

$$
q_{1}^{K V}=\frac{a(b+c)}{b(3 b+2 c)} ; \quad q_{2}^{K V}=\frac{a}{3 b+2 c} \text { and } p^{K V}=\frac{a(b+c)}{3 b+2 c},
$$

with profits,

$$
\pi_{1}^{K V}=\frac{a^{2}(b+c)^{2}}{b(3 b+2 c)^{2}}-F \text { and } \pi_{2}^{K V}=\frac{a^{2}(2 b+c)}{2(3 b+2 c)^{2}} .
$$

It is straightforward to see that $\pi_{1}^{V V} \geq \pi_{1}^{K V}$, namely that choosing $V$ against firm 2 choosing $V$ is optimal for firm 1 if, and only if

$$
F \geq F^{I}
$$

where $F^{I}>0 .^{5}$

By symmetry, condition (10) guarantees that the best reply of firm 2 against $V$ is $V$ itself. We summarize this result in the following

Lemma 2 Let $F^{I}<F$. Then at the unique SPNE of the game $\Gamma$ both firms choose the decreasing returns to scale technology $V$ and equilibrium price is $p^{V V}=a(b+c) /(3 b+c)$.

Easy calculations show that

$$
F^{I}<F^{I I} \forall\{a, b, c\}>0 .
$$

We do not describe in full the other possible equilibria, for lower values of $F$. There are parameter regions where one firm chooses $Y^{K}$ and the other $Y^{V}$, and parameter regions where both choose $Y^{K}$. The main result deriving from our example can be stated as follows:

Proposition 1 (i) If $F^{I}<F<F^{I I}$ and $b<c$, then entry by one firm into the monopolized industry leads to an increase in industry price.

(ii) If $F<F^{I}$ entry entry by one firm into the monopolized industry leads to a lower price.

\footnotetext{
${ }^{4}$ The notation $K V$ stands for "firm 1 selects $K$ and firm 2 selects $V$ ". ${ }^{5} F^{I}=\frac{a^{2} c\left(15 b^{3}+24 b^{2} c+12 b c^{2}+2 c^{3}\right)}{2 b(3 b+c)^{2}(3 b+2 c)^{2}}$.
} 


\section{Proof.}

(i) If $F^{I}<F<F^{I I}$, by Lemma 1 we know that the monopolist selects technology $K$, with equilibrium price $p_{M}^{K}$, and by Lemma 2 both duopolists choose $V$, with equilibrium price $p^{V V}$. The difference $p_{M}^{K}-p^{V V}=\frac{a(b-c)}{2(3 b+c)}$ is negative if and only if $b<c$.

(ii) If $F<F^{I}$, after entry at least one firm chooses the $K$-technology. It can be checked that the duopoly equilibrium price always falls short $\frac{a}{2}=p_{M}^{K}$, the monopoly one.

The intuition behind part (i) of Proposition 1 is as follows. Entry leads the incumbent to reduce the quantity it supplies to the market, in response to the decrease in its residual demand. This standard effect positively affects prices. In our case, in addition, the reduction in the incumbent's quantity is accompanied by a shift from the increasing returns to scale technologywhich is, from the single firm's standpoint, better suited for large-scale production - to the decreasing returns to scale one - more efficient at low production regimes. This technological change, allowed by the assumption of technical flexibility, increases the marginal production costs of the incumbent, reducing even further its optimal output level. If the industry's features are such that the entrant chooses the $V$ technology as well, the quantity it supplies to consumers fails to compensate the reduction operated by firm 1 .

Part (ii) of Proposition 1 is (standardly) explained by the combination of increased competition with possibly lower marginal production costs.

\section{Conclusion}

The result stated in part (i) of Proposition 1 is the core result of our example: using specifications of production sets commonly adopted in the economic literature we proved that letting an incumbent react to entry by modifying both the quantity of good supplied to the market and the manner that good is produced can result in an increase in equilibrium price. 
Notwithstanding this, there are parameter regions in which the example is quasi-competitive, so that entry lowers the market price (part (ii) of Proposition 1); these regions correspond to situations in which, at equilibrium, the incumbent does not abandon the $K$ technology in response to entry. ${ }^{6}$ Moreover it can be easily checked that if the incumbent were committed to the technology chosen when enjoying monopoly power, entry would always lower prices, so that our example would display the quasicompetitiveness property.

These observations strengthen our initial claim that the assessment on the benefits from competition may be distorted by the assumption of technical inflexibility. Indeed the option to change the production technology and the decision to exercise this option prove to be crucial for observing a market price increase after entry. As a consequence, the time dimension emerges as another central feature for these issues: the long run effects of entry on prices, when firms are technically flexible by definition, may have opposite sign than those in the short run, when firms are subject to (some degree of) technical inflexibility. The role played by different marginal costs is crucial as well: their increase following the shift in technology, even if optimal from the firms' viewpoint, translates into a decrease in quantity, and hence an increase in market price, which reduces consumers' surplus.

\section{References}

[1] Amir, R. and Lambson, V.E. (2000). On the Effects of Entry in Cournot Markets, Review of Economic Studies, 67, 235-254.

[2] Frank, C.R. (1965). Entry in a Cournot Market, Review of Economic Studies, 32, 245-250.

[3] Okuguchi, K. (1973). Quasi-Competitiveness and Cournot Oligopoly, Review of Economic Studies, 40, 145-148.

[4] MacManus, M. (1962), Number and Size in Cournot Equilibrium, Yorkshire Bulletin of Economic and Social Research, 14, 14-22.

\footnotetext{
${ }^{6} \mathrm{Or}$, if the incumbent abandons $Y^{K}$, the entrant selects it, leading to at least one firm using technology $K$.
} 
[5] MacManus, M. (1964), Equilibrium, Number and Size in Cournot Oligopoly, Yorkshire Bulletin of Economic and Social Research, 16, 6875 .

[6] Ruffin, R.J. (1971). Cournot Oligopoly and Competitive Behavior, Review of Economic Studies, 38, 493-502.

[7] Seade, J. (1980). On the Effects of Entry, Econometrica, 48, 479-489. 